\title{
Electrostatic up-element and promoter strength
}

\author{
A. Osypov ${ }^{1,2 *}$, G. Krutinin 3 , E. Krutinina3 3 , P. Beskaravayny ${ }^{3}$, S. Kamzolova ${ }^{3}$ \\ ${ }^{1}$ Institute of Higher Nervous Activity and Neurophysiology RAS, Moscow, Russia \\ ${ }^{2}$ Institute of Theoretical and Experimental Biophysics RAS, Pushchino Moscow Region, Russia \\ ${ }^{3}$ Institute of Cell Biophysics RAS, Pushchino Moscow Region, Russia \\ *e-mail:aosypov@gmail.com
}

Key words: DNA electrostatics, transcription regulation, genome evolution

Motivation and Aim: It is known that not only the consensus sequence text is essential for RNA polymerase-promoter recognition and regulation, but physical properties, especially electrostatics, play important role particularly at the early stages of this process. One of the elements that may play a crucial role in the promoter strength regulation is a so-called "up-element", which interacts with the alpha-subunit of RNAP and thus facilitates its binding to the promoter. There is no text consensus in the "up-element" (though high AT content is often attributed) and functionality of this region is defined by its physical properties. We have shown earlier, that electrostatics is responsible for its functioning during the global transcription switch under the T4 bacteriophage infection and that strong T4, early T7-like, phage Lambda and E. coli ribosomal promoters with pronounced up-element have high levels of the electrostatic potential within it.

Methods and Algorithms: DEPPDB and its tools [1,2] were used to carry out the analysis. Mutant promoters' sequences and strength are taken from $[3,4]$.

Results: In the strong E. coli ribosomal rrnB P1 promoter and its up-element mutants the promoter strength depends upon the size of the electrostatic up-element so that the bigger the element - the stronger the promoter is. However, if the element is too big the strength decreases slightly due to possible trapping of the polymerase that hinders the transcription initiation or elongation.

Conclusion: Electrostatics may play important role in the transcription regulation in the first step of initial promoter recognition and the second step of the transcription initiation due to interactions between the RNA-polymerase alpha subunit and the promoter upelement that has electrostatic nature.

Acknowledgements: Supported by the RFBR (16-04-01865 and 18-34-00942).

\section{References}

1. Osypov A.A. et al. (2010) DEPPDB - DNA Electrostatic Potential Properties Database: Electrostatic properties of genome DNA. JBCB. 8(3):413-425.

2. Osypov A.A. et al. (2012) DEPPDB - DNA Electrostatic Potential Properties Database: Electrostatic properties of genome DNA elements. JBCB. 10(2):1241004.

3. Estrem S.T. et al. (1998) Identification of an UP element consensus sequence for bacterial promoters. Proc. Natl. Acad. Sci. USA. 95(17):9761-9766.

4. Estrem S.T. et al. (1999) Bacterial promoter architecture: subsite structure of UP elements and interactions with the carboxy-terminal domain of the RNA polymerase alpha subunit. Genes Dev. 13:2134-2147. 